\title{
Comparison of Two Distraction Devices for Assessment of Passive Hip Laxity in Dogs
}

\author{
Ana Santana ${ }^{1,2}$, Sofia Alves-Pimenta ${ }^{2,3}$, João Martins ${ }^{1,2}$, Bruno Colaço $^{2,3}$ and \\ Mário Ginja ${ }^{2,4 *}$
}

\begin{abstract}
${ }^{1}$ Faculty of Veterinary Medicine, Lusófona University, Lisbon, Portugal, ${ }^{2}$ CITAB-Centre for the Research and Technology of Agro-Environmental and Biological Sciences, University of Trás-os-Montes and Alto Douro, Vila Real, Portugal, ${ }^{3}$ Department of Animal Science, University of Trás-os-Montes and Alto Douro, Vila Real, Portugal, ${ }^{4}$ Department of Veterinary Science, University of Trás-os-Montes and Alto Douro, Vila Real, Portugal
\end{abstract}

\section{OPEN ACCESS}

Edited by:

Susanne M. Stieger-Vanegas, Oregon State University, United States

Reviewed by: Tommaso Banzato, University of Padua, Italy Jennifer Juliet Warnock

Oregon State University, United States Jean-Pierre Genevois, VetAgro Sup, France

*Correspondence: Mário Ginja mginja@utad.pt

Specialty section: This article was submitted to Veterinary Imaging, a section of the journal

Frontiers in Veterinary Science

Received: 30 January 2020

Accepted: 30 June 2020

Published: 19 August 2020

Citation

Santana A, Alves-Pimenta S, Martins J, Colaço $B$ and Ginja M (2020) Comparison of Two Distraction

Devices for Assessment of Passive Hip Laxity in Dogs.

Front. Vet. Sci. 7:491 doi: 10.3389/fvets.2020.00491
Canine hip dysplasia is the most common orthopedic developmental condition in the dog and early hip laxity is the main risk factor. The importance of hip laxity in young animals in the development of hip dysplasia is unanimously recognized among researchers and veterinarians due to its medical applicability in terms of disease control and prevention. In the market, there is some certified hip distractors to promote joint laxity. However, the clinical use of some of these distractors complies with a set of usage rules, that can limit its medical application. In this study was compared the technical quality of radiographs and hip distraction using a certified hip distractor (CertD) and Dis-UTAD in 104 dogs (208 joints). The mean pelvic tilting of $1.5 \pm 1.6^{\circ}$ and $1.5 \pm 1.8^{\circ}$ were similar when using the CertD and the Dis-UTAD distractors, respectively $(P>0.05)$. In the CertD sample, the mean hip distraction index (DI) was $0.46 \pm 0.17$ and in the Dis-UTAD $0.46 \pm 0.16$; the mean DI differences was $0.001 \pm 0.045$, resulting in a non-significant paired $t$-test $(P=0.65)$ and a significant intraclass correlation coefficient of 0.96 , with the $95 \%$ lower limit confidence interval of $0.95(P<0.05)$. The statistical power analysis showed a very low distraction index difference effect size. The results suggest that the statistical reproducibility of CertD hip distraction by the Dis-UTAD and the DI mean differences of 0.001 might be considered without clinical importance. The Dis-UTAD might be considered adequate to promote dog hip laxity.

Keywords: hip laxity, distraction index, reproducibility, PennHip, Dis-UTAD, dog

\section{INTRODUCTION}

Canine hip dysplasia (CHD) is a complex, inherited, polygenic trait disease influenced by multiple environmental factors, which was first identified in dogs by Schnelle in 1935 (1-3). CHD is considered as one of the most common orthopedic developmental conditions in dogs that lead to a debilitating secondary hip osteoarthritis (4). Although the etiology of CHD is not completely understood, increased laxity of the hip joint is the most frequent early cause reported and usually results in secondary osteoarthritis (OA) (5). CHD is a challenging disease to prevent, diagnose, and manage. Clinical signs such as decreased activity, difficulty in rising, "bunny hopping" gait, hind limb lameness, and hip pain support the suspicion of the disease (6). The actual diagnosis is confirmed radiographically if characteristic signs are evident on standard hip extended view (HEV) 
in dogs over 1 year of age (4). There is not an adequate molecular diagnosis for hip dysplasia (3); therefore, radiographic diagnosis has been essential for the selection of breeding stock and is based on two main key features: the detection of signs of degenerative joint disease or the diagnosis of early hip joint laxity $(\mathrm{HJL})(7,8)$. Although HEV has been shown to be a valuable tool in evaluating the presence of OA, it can severely underestimate HJL because of the non-physiological tensioning of the pelvic muscles and twisting of the joint capsule (5). Distraction-stress radiography techniques are used to better estimate the degree of passive HJL through the calculation of distraction index (DI) $(5,9)$. In the hip distraction view, the femoral heads are displaced laterally by the use of a custom-designed device (distractor) placed between the legs that acts as a fulcrum on the femur at the level of the ventral aspect of the pelvis (5). The DI is obtained by dividing the lateral femoral head displacement by its radius; a DI of 0 represents absolute joint congruity and a DI of 1 represents complete joint luxation (5). The hip joint distractors PennHIP $(5,7)$ and "FSA-Fondazione Salute Animale" (9) have been used in published works to obtain hip distraction views.

The purpose of the present study was to compare the technical quality of radiographs and hip distraction using the CertD and the Dis-UTAD, a hip distractor developed at the University of Trás os Montes e Alto Douro (UTAD).

\section{MATERIALS AND METHODS}

\section{Animals}

In this prospective study, 104 dogs (58 females and 46 males) from five different breeds (68 Estrela Mountain dogs, 12 Transmontano Cattle Dog, 12 Portuguese Pointer Dog, 11 Rafeiro do Alentejo, and one Barbado da Terceira) were presented at the Veterinary Teaching Hospitals of University Lusófona de Humanidades e Tecnologias and University of Trás-os-Montes and Alto Douro between the years of 2014 and 2019 and screened for passive hip laxity. The recorded data included breed, age at the time of radiography, sex, and body weight. The inclusion criteria were that dogs had to be from Portuguese breeds, between 4 and 12 months of age at the time of the exam, and presenting normal musculoskeletal development upon clinical examination. The minimum sample size was estimated using a $t$-test table and selecting a statistical significance of 0.05 , a medium variable effect size of 0.4 , and a statistical power of 0.8 , which indicated a sample of 99 observations (10).

All the radiographic examinations were performed with the dog owner's consent and all the animal procedures were undertaken as part of the work described in this study, performed in compliance with the regulations of our institutions (no. 1044-e-DCV-2018) and in accordance with the Portuguese and European regulations for animal use and care (European Directive 2010/63/EU and National Decree-Law 113/2013).

\section{Radiographic Procedure}

The radiographs were performed with the dogs under deep sedation using medetomidine (Domitor: Orion Corporation, Espoo, Finland) and butorphanol (Torbugesic Injectable: Fort Dodge Veterinaria, Girona, Spain) administered intravenously. The sedation was reversed with atipamezole hydrochloride (Antisedan: Orion Corporation, Espoo, Finland) intramuscularly. Radiographs were obtained in the same sequence with the dogs in dorsal recumbency on the X-ray table: first the HEV and then two distraction views with the distractor device placed between the hind limbs to promote passive hip laxity, first using the CertD and followed by the second distraction view using the Dis-UTAD. The Dis-UTAD is a modified Vezzoni distension device with an isosceles trapezoid shape (9); it has an external rubber component and a polyethylene plate in the interior that gives it longitudinal flexibility and transverse stiffness (11). With the dog in dorsal recumbency, hip distraction was achieved in a similar way to the PennHIP and Vezzoni techniques $(5,9)$. Both femurs were adducted by the examiner symmetrically in a neutral position $(+/-10$ degrees) against the distractor, fixed on the animal with the support of two cylindrical sandbags weighing $\sim 4 \mathrm{~kg}$ each, one at the front and one at the distractor's back (Figure 1). On the distraction radiograph, the pelvis and the distractor should appear centered and symmetrical, and the more pronounced lateral band opacity of the distractor overlaps the femoral heads (Figure 2). The hip distractor fixation with sandbags was already described previously to avoid the exposure of the examiner's assistant to ionizing radiation (12). The radiographs were taken by veterinarians with experience in hip stress views (AS and MG).

\section{Positioning Evaluation and Hip Laxity Measurement}

Technical radiographic positioning analysis was performed by estimating the grade of pelvis tilting $(y)$ and measuring the asymmetry of the obturator foramen width (OFW) $(x), y=1.6 x$ - 0.9 (13). For the hip laxity measurements, a dedicated semiautomatic software was used as previously described (14). The DI was calculated by dividing the distance between the centers of the femoral head and the acetabulum by the radius of the femoral head, as described previously (5). Both measurements of pelvic tilting and DI were performed in two independent sessions by JM and AS, respectively.

\section{Statistical Analysis}

Statistical analysis was performed using the computer software SPSS (SPSS Statistics for Windows Version 23.0: IBM Corp., Armonk, NY, USA). The data analysis was performed on joints individually. The paired $t$-test and the intraclass correlation coefficient (ICC) were used in comparing the pelvic tilting and the DI of both hip distractors in order to evaluate Dis-UTAD's reproducibility $(15,16)$. A value of $p<0.05$ was considered to be statistically significant. The null hypothesis was that the mean difference between paired observations was zero (10). The size effect and the statistical power were estimated to evaluate the ability of our sample to detect variable differences on each distractor set (10).

\section{RESULTS}

The dogs' age ranged from 4 to 11 months (mean \pm standard deviation, $6.0 \pm 2.1$ months), and body weight ranged from 13.5 to $54 \mathrm{~kg}$ (mean, $24.7 \pm 8.6 \mathrm{~kg}$ ). In the CertD sample, 
A

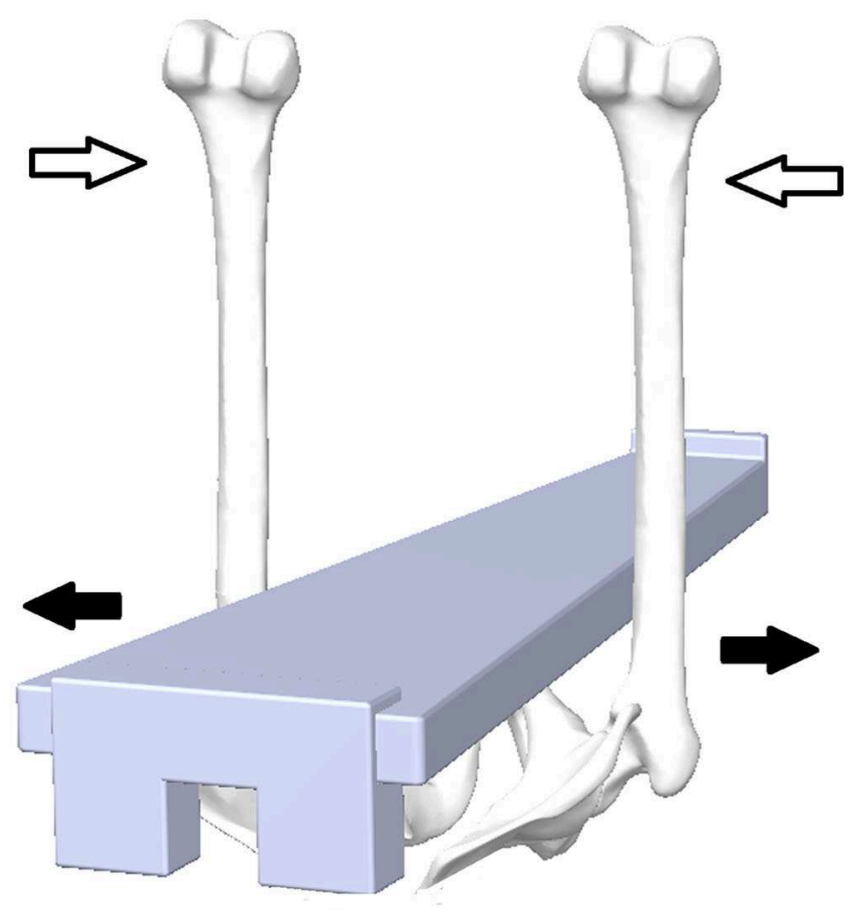

B

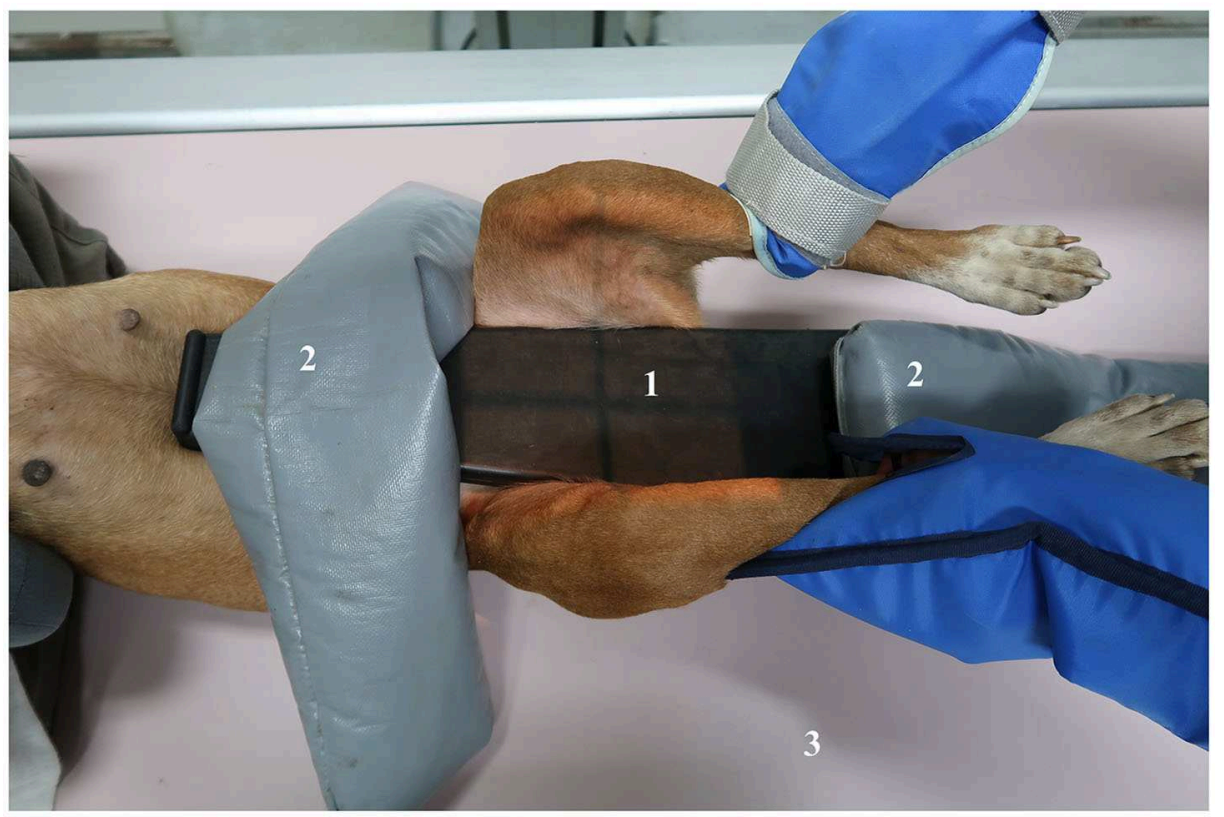

FIGURE 1 | The Dis-UTAD is a modified Vezzoni distension device. (A) Illustration outlining the rear view of the dog and the Dis-UTAD. The open arrows represent the medial force exerted by the examiner, pushing the femurs against the distractor, and the full arrows represent the resulting hip distraction force. (B) Dis-UTAD positioned on the animal, fixed by the support of two sandbags. 1, Dis-UTAD; 2, sandbags; 3, X-ray table.

the pelvic tilting ranged from 0 to $6.5^{\circ}$ (mean, $1.5 \pm 1.6^{\circ}$ ) and the DI ranged from 0.16 to 0.88 (mean, $0.46 \pm 0.17$ ). In radiographs obtained with the Dis-UTAD, pelvic tilting ranged from 0 to $6.3^{\circ}$ (mean, $1.5 \pm 1.8^{\circ}$ ) and the DI ranged from 0.12 to 0.88 (mean $0.46 \pm 0.16$ ). Comparing CertD and Dis-UTAD, for pelvic tilting the mean of the difference was $0.04 \pm 1.9^{\circ}$ and for DI it was $0.001 \pm 0.045^{\circ}$, and the paired $t$-test was not statistically significant in both evaluations, being $P=0.84$ 
and 0.65 , respectively (Table 1 and Figure 3). The ICC between both DI samples for single measures was 0.96 (95\% confidence interval, 0.95-0.97).

\section{DISCUSSION}

Hip joint laxity is the main risk factor for the development of degenerative joint disease in dogs and is associated with high heritability $(3,8)$. Therefore, the use of DI breeding selection is highly recommended in the control programs of CHD (4). The clinical achievement of the distraction view requires some specific training and PennHIP has free online courses available. However, the clinical use of some hip distractors complies with a set of imposed rules that can limit its clinical usage $(9,17)$. The Dis-UTAD was developed to overcome some restrictions in hip distractor availability and intended, for interested veterinarians, as an adequate alternative in the assessment of dog's hip joint laxity. However, like the usage of other hip distractors, a previous practical training is recommended to perform adequate hip distraction views as well as for the distraction index measurement (14).

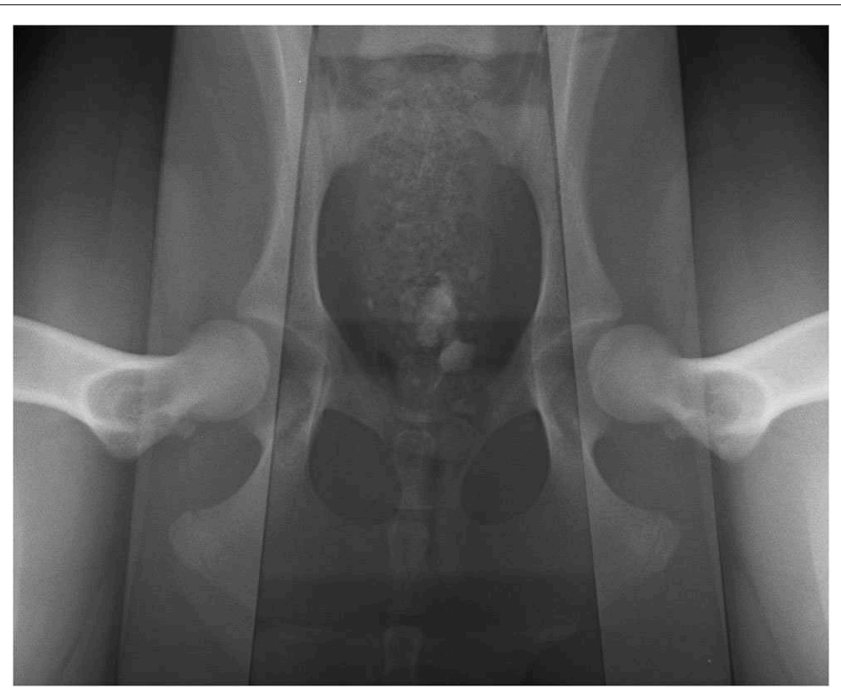

FIGURE 2 | Hip distraction view using the Dis-UTAD distractor. The pelvis and the distractor are centered and symmetrical, and the more pronounced lateral band opacity of the distractor overlaps the femoral heads.
In this work, the OFW was used to evaluate the grade of pelvis tilting and not the iliac horizontal diameter as recommended in a previous work that used the HEV (13) because, in some radiographs of our sample, the X-ray collimation did not allow the observation of all sacroiliac joints. Nonetheless, this study showed also a good correlation between OFW and pelvic tilting (13). Our work shows that the degree of pelvic tilting using Dis-UTAD is similar to the one that we obtain by using the CertD. The mean degree of pelvic tilting in our samples $\left(1.5^{\circ}\right)$ was slightly higher than those in other works where conventional hip ventrodorsal view was used $(13,18)$. There are no previous published distraction hip studies where the degree of pelvic rotation has been evaluated. Theoretically, the tilting of the pelvis should not have much interference in DI measurement since this variable results from two spherical anatomical structures in a similar dorsal dog anatomical plane and relatively close to the center of the X-ray beam. Radiographic spatial distortion is especially important in the periphery of the X-ray beam and when the reference structures are at different distances from the radiographic film (19).

The non-significant $t$-test and the ICC of 0.96 with a lower limit of the $95 \% \mathrm{CI} \geq 0.75$ indicate that there is no bias, a strong association between the hip distraction promoted by both distractors and statistical reproducibility and interchangeability (16). However, the low statistical test power does not allow us to reject the false null hypothesis (10). As the mean DI difference effect size is very low (0.07), we will need a sample of more than 1,500 animals to obtain enough statistical power $(0.80)$ to demonstrate that the DI differences are not due to the Dis-UTAD distractor (10). However, when the mean variable differences are exceedingly small, they can be considered without medical importance $(20,21)$. There is no statistical test powerful enough to detect variable differences between samples with a mean of 0 since an infinite sample would be needed for comparison (21). The DI measurement differences included also examiner and scoring errors (22), which are difficult to differentiate. A similar reproducibility of the PennHIP method results was obtained in a recent study using the Vezzoni Modified Badertscher distractor (9). The longitudinal flexibility of the DisUTAD allows good adaptation to the dog's body and the sandbags stabilize it adequately, resisting better to the examiner's medial force on the hind limbs. Distractor stabilization is also needed in the PennHIP distractor using sandbags (12) or the help of an assistant (5), which we do not recommend because it increases human exposure to ionizing radiation.

TABLE 1 | Paired variable differences between CertD and Dis-UTAD.

\begin{tabular}{|c|c|c|c|c|c|c|c|c|c|c|}
\hline \multirow[t]{2}{*}{ Variable } & \multirow[t]{2}{*}{$N$} & \multicolumn{6}{|c|}{ Paired differences } & \multirow[t]{2}{*}{$r$} & \multirow[t]{2}{*}{ Effect size } & \multirow[t]{2}{*}{ Power } \\
\hline & & Mean & SD & SEM & Lower & Upper & $P$ & & & \\
\hline PT $\left(^{\circ}\right)$ & 104 & -0.04 & 1.87 & 0.18 & -0.4 & 0.33 & $>0.05$ & 0.4 & 0.02 & 0.05 \\
\hline DI & 208 & 0.001 & 0.045 & 0.003 & -0.005 & 0.008 & $>0.05$ & 0.96 & 0.03 & 0.07 \\
\hline
\end{tabular}

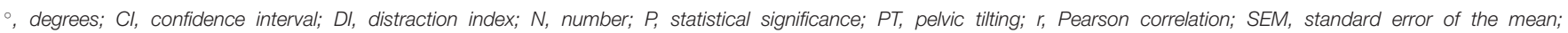
$S D$, standard deviation. 


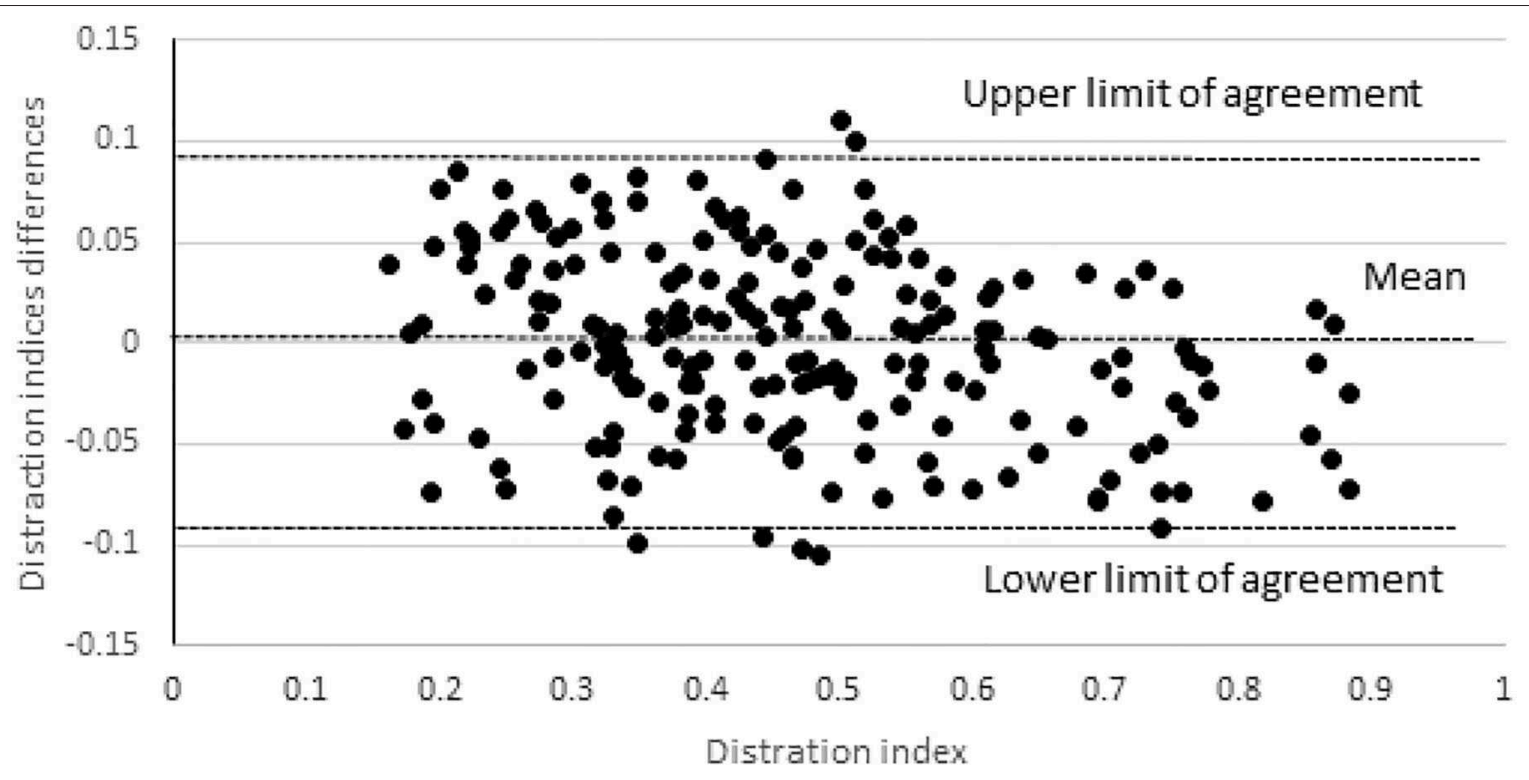

FIGURE 3 | Differences between the distraction index obtained in distraction radiographs using the certified distractor and the Dis-UTAD. The horizontal lines represent the mean of the differences (0.001) and the upper and the lower 95\% limits of agreement, $\sim 0.09$ and -0.09 , respectively.

The higher lateral band opacity of the distractor, overlying the femoral heads, gives the examiner a good idea of the symmetry of positioning and distraction level (Figure 2). It is recommended to repeat the examination when this does not happen. The rear Dis-UTAD thicker component with a central hole allows table support, accommodates the tail of the dog, and allows a more horizontal use of the distractor, characteristics that facilitate the hip distraction process. The heterogeneity of our sample (animals of about $10-50 \mathrm{~kg}$ ) also shows that Dis-UTAD has the ability to promote adequate hip distraction in small and large dogs using its cranial or caudal part in distraction, respectively. Nonetheless, the small number of breeds can be pointed out as a limitation of this study. The DI ICC in this study (0.96) was higher than the within- (0.94) and between-examiner (0.91) DI repeatability of previous studies using the PennHIP distractor (17) and similar to other studies that evaluate the reproducibility of PennHIP DI measurements (22). These facts may be associated with the examiner's expertise and the reliability of the DI measurement method or may be related to the size or the type of the sample used. The dedicated semiautomatic software used in hip laxity measurements was already used in a previous work, which proved to be effective (14).

This and other recent studies show that joint laxity can be reliably quantified with the use of different distractors and methods (9), and there are scientific and technical conditions for extending their use in breeding selection and for preventive CHD management purposes. The recognition of the importance of hip laxity in young animals in the development of hip dysplasia is unanimous among researchers and veterinarians $(5,7,9,23$, $24)$, so its medical applicability in terms of disease control and prevention should be promoted between veterinarians, owners, and dog breeders. However, it should be kept in mind that the success of hip dysplasia control programs depends more on the knowledge of the breeding population than on the dog alone, and databases with reliable medical information are essential $(25,26)$.

\section{CONCLUSIONS}

Pelvic tilting and DI measured using the Dis-UTAD showed the statistical reproducibility of the CertD measurements. The mean DI difference of 0.001 might be considered without clinical importance. The Dis-UTAD might be considered adequate to promote dog hip laxity.

\section{DATA AVAILABILITY STATEMENT}

All datasets generated for this study are included in the article/Supplementary Material.

\section{ETHICS STATEMENT}

The animal study was reviewed and approved by Comissão de Ética da Universidade de Trás-os-Montes e Alto Douro (CE-UTAD). Written informed consent was obtained from the owners for the participation of their animals in this study.

\section{AUTHOR CONTRIBUTIONS}

AS contributed to the acquisition of data and drafting of the manuscript. SA-P contributed to the critical revision of the manuscript. JM contributed to the acquisition of data and critical revision of the manuscript. $\mathrm{BC}$ contributed to the concept/design and critical revision of manuscript. MG contributed to the 
concept/design, acquisition of data, data analysis/interpretation, and drafting of the manuscript. All authors contributed to the article and approved the submitted version.

\section{ACKNOWLEDGMENTS}

This work was supported by the National Funds by FCTPortuguese Foundation for Science and Technology, under

\section{REFERENCES}

1. Henricson B, Norberg I, Olsson SE. On the etiology and pathogenesis of hip dysplasia: a comparative review. J Small Anim Pract. (1966) 7:673-88. doi: 10.1111/j.1748-5827.1966.tb04393.x

2. King MD. Etiopathogenesis of canine hip dysplasia, prevalence and genetics. Vet Clin N Am Small Anim Pract. (2017) 47:753-67. doi: 10.1016/j.cvsm.2017.03.001

3. Ginja M, Gaspar AR, Ginja C. Emerging insights into the genetic basis of canine hip dysplasia. Vet Med (Auckl). (2015) 6:193-202. doi: 10.2147/VMRR.S63536

4. Ginja MMD, Silvestre AM, Gonzalo-Orden JM, Ferreira AJA. Diagnosis, genetic control and preventive management of canine hip dysplasia: a review. Vet J. (2010) 184:269-76. doi: 10.1016/j.tvjl.2009.04.009

5. Smith GK, Biery DN, Gregor TP. New concepts of coxofemoral joint stability and the development of a clinical stress-radiographic method for quantitating hip joint laxity in the dog. J Am Vet Med Assoc. (1990) 196:59-70.

6. Fries CL, Remedios AM. The pathogenesis and diagnosis of canine hip dysplasia: a review. Can Vet J. (1995) 36:494-502.

7. Ginja MM, Ferreira AJ, Jesus SS, Melo-Pinto P, Bulas-Cruz J, Orden MA, et al. Comparison of clinical, radiographic, computed tomographic, and magnetic resonance imaging methods for early prediction of canine hip laxity and dysplasia. Vet Radiol Ultrasound. (2009) 50:135-43. doi: 10.1111/j.1740-8261.2009.01506.x

8. Smith GK, Popovitch CA, Gregor TP, Shofer FS. Evaluation of risk factors for degenerative joint disease associated with hip dysplasia in dogs. J Am Vet Med Assoc. (1995) 206:642-7.

9. Broeckx BJ, Vezzoni A, Bogaerts E, Bertal M, Bosmans T, Stock E, et al. Comparison of three methods to quantify laxity in the canine hip joint. Vet Comp Orthop Traumatol. (2018) 31:23-9. doi: 10.3415/VCOT17-05-0064

10. Cohen J. Statistical Power Analysis for the Behavioral Sciences. 2nd ed. (1988). London: Lawrence Erlbaum Associates, Inc.

11. Ginja M. University of Trás-os-Montes and Alto Douro. Dis-UTAD hip distractor. Portuguese patente $n^{\circ} 107372$. Vila Real; Lisbon: Instituto Nacional da Propriedade Industrial (INPI) (2017).

12. Ginja MM, Llorens-Pena MP, Gonzalo-Orden JM, Ferreira AJ. Mechanical devices to help in PennHIP examination. Acta Vet Hung. (2007) 55:199-205. doi: 10.1556/avet.55.2007.2.5

13. Martins J, Colaço BJ, Ferreira AJ, Ginja MM. Analysis of pelvic rotation on the standard hip ventrodorsal extended radiographic view. Vet Comp Orthop Traumatol. (2016) 9:68-74. doi: 10.3415/VCOT-15-02-0025

14. Alves-Pimenta S, Santana A, Martins J, Colaço B, Gonçalves L, Ginja M. Anatomical parameters measured on the dog's hip joint using a dedicated software. Arq Bras Med Vet Zootec. (2020) 72:1241-7.

15. Petrie A, Watson P. Statistics for Veterinary and Animal Science. Oxford: Blackwell Science (1999).

16. Lee J, Koh D, Ong CN. Statistical evaluations of agreement between two methods for measuring a quantitative variable. the projects UIDB/04033/2020 and Scientific Employment Stimulus-Institutional Call-CEECINST/00127/2018 UTAD.

\section{SUPPLEMENTARY MATERIAL}

The Supplementary Material for this article can be found online at: https://www.frontiersin.org/articles/10.3389/fvets. 2020.00491/full\#supplementary-material

Comput Biol Med. (1989) 19:61-70. doi: 10.1016/0010-4825(89) 90036-X

17. Ginja MM, Ferreira AJ, Silvestre M, Gonzalo-Orden JM, Llorens-Pena MP. Repeatability and reproducibility of distraction indices in PennHIP examinations of the hip joint in dogs. Acta Vet Hung. (2006) 54:387-92. doi: 10.1556/avet.54.2006.3.8

18. Martins J, Colaço B, Alves-Pimenta S, Gonzalo Orden JM, Ferreira AJ, Ginja MM. Effect of the dog positioning on X-ray table on hip dysplasia parameter evaluation. Vet Comp Orthop Traumatol. (2019) 32:376-82. doi: 10.1055/s-0039-1688991

19. Mixdorf M, Tortorici M. Geometric image quality. In: Tortorici M, editor. Concepts in Medical Radiographic Imaging: Circuitry, Exposure and Quality Control. Philadelphia, PA: WB Saunders (1992). p. 310-23.

20. Jones SR, Carley S, Harrison M. An introduction to power and sample size estimation. Emerg Med J. (2003) 20:453-8. doi: 10.1136/emj. 20.5.453

21. Santana A, Alves-Pimenta S, Martins J, Colaço B, Ginja M. Hands-free conventional radiographic ventrodorsal hip extended view. Front Vet Sci. (2020) 6:378. doi: 10.3389/fvets.2020.00286

22. Smith GK, LaFond E, Gregor TP, Lawler DF, Nie RC. Within- and betweenexaminer repeatability of distraction indices of the hip joints in dogs. Am J Vet Res. (1997) 58:1076-7.

23. Farese JP, Todhunter RJ, Lust G, Williams AJ, Dykes NL. Dorsolateral subluxation of hip joints in dogs measured in a weight-bearing position with radiography and computed tomography. Vet Surg. (1998) 27:393-405. doi: 10.1111/j.1532-950X.1998.tb00146.x

24. Flückiger M, Friedrich GA, Binder H. A radiographic stress technique for evaluation of coxofemoral joint laxity in dogs. Vet Surg. (1999) 28:1-9. doi: 10.1053 /jvet.1999.0001

25. Ohlerth S, Geiser B, Flückiger M, Geissbühler U. Prevalence of canine hip dysplasia in Switzerland between 1995 and 2016-a retrospective study in 5 common large breeds. Front Vet Sci. (2019) 6:378. doi: $10.3389 /$ fvets. 2019.00378

26. James HK, McDonnell F, Lewis TW. Effectiveness of canine hip dysplasia and elbow dysplasia improvement programs in six UK pedigree breeds. Front Vet Sci. (2020) 6:490. doi: 10.3389/fvets.2019.00490

Conflict of Interest: The authors declare that the research was conducted in the absence of any commercial or financial relationships that could be construed as a potential conflict of interest.

Copyright (C) 2020 Santana, Alves-Pimenta, Martins, Colaço and Ginja. This is an open-access article distributed under the terms of the Creative Commons Attribution License (CC BY). The use, distribution or reproduction in other forums is permitted, provided the original author(s) and the copyright owner(s) are credited and that the original publication in this journal is cited, in accordance with accepted academic practice. No use, distribution or reproduction is permitted which does not comply with these terms. 